\title{
Klimaschutz im Luftverkehr: vom EU-Emissionshandel zu CORSIA
}

\author{
Als ein Baustein zur Erreichung des Ziels eines $\mathrm{CO}_{2}$-freien Wachstums des internationalen \\ Luftverkehrs hat die Generalversammlung der Internationalen Zivilluftfahrtorganisation \\ die Einführung der marktbasierten Klimaschutzmaßnahme CORSIA ab 2020 beschlossen. \\ CORSIA wird auch im Vergleich zum bislang in Europa auf den Luftverkehr angewendeten \\ Emissionshandelssystem EU-ETS vorgestellt. Das neue umweltpolitische Instrument wird \\ anschließend sowohl aus umwelt- als auch aus transaktionskosten-ökonomischer und \\ wettbewerbspolitischer Sicht für den Passagierluftverkehr kritisch gewürdigt.
}

Der weltweite Luftverkehr hatte bereits 2005 einen geschätzten Anteil von rund $2,5 \%$ am gesamten, vom Menschen verursachten $\mathrm{CO}_{2}$-Ausstoß. Berücksichtigt man weitere Faktoren wie etwa die Emission von Stickoxiden, die zu Ozonbildung und Methanabbau führen, erhöht sich der Gesamtbeitrag des Luftverkehrs am menschengemachten Klimawandel auf rund 4,9\%. ${ }^{1}$

Um den Klimabeitrag der $\mathrm{CO}_{2}$-Emissionen des Wachstumssektors Luftverkehr zu senken, wurde dieser 2012 in den EU-Emissionshandel (EU-ETS) einbezogen. Eine Maßnahme, die aufgrund massiver Vorbehalte vieler Drittstaaten aber entgegen ursprünglicher Planungen bis heute nur auf Flüge innerhalb des Europäischen Wirtschaftsraums (EWR) angewendet wird und daher in ihrer Wirkung begrenzt ist. Um das 2010 in Resolution A37-19 der International Civil Aviation Organization (ICAO) formulierte Ziel eines $\mathrm{CO}_{2}$-freien Luftverkehrswachstums ab 2020 (Carbon Neutral Growth 2020, kurz: CNG2020) dennoch zu erreichen, wurde im Herbst 2016 in ICAO-Resolution A39-3 erstmals eine marktbasierte Klimaschutzmaßnahme für den globalen Luftverkehr beschlossen. Sie trägt die Bezeichnung CORSIA (Carbon Offsetting and Reduction Scheme for International Aviation) und sieht eine geografisch wesentlich breitere Abdeckung vor als das EU-ETS.

\section{Marktbasierte Maßnahmen - ETS versus Offsetting}

Bei $\mathrm{CO}_{2}$-Emissionen handelt es sich um negative externe Effekte, für deren Regulierung zwischen ordnungsrechtlichen Instrumenten wie Auflagen und Normen einerseits und umweltökonomischen Ansätzen andererseits unter-

1 Vgl. D. S. Lee et al.: Aviation and global climate change in the 21st century, in: Atmospheric Environment, 43. Jg. (2009), H. 22-23, S. $3520-3537$. schieden wird. Zu letzteren zählen neben (Pigou-)Steuer-, Abgaben- und Subventionslösungen auch Second-BestAnsätze wie Zertifikats- und Standard-Preis-Lösungen. ${ }^{2}$ Sowohl das 2005 für stationäre Anlagen und später auch für den innereuropäischen Luftverkehr eingeführte Emissionshandelssystem (Cap\&Trade-System) EU-ETS als auch das angekündigte Klimakompensationssystem CORSIA sind Beispiele für Zertifikatslösungen.

Im Rahmen eines Cap\&Trade-Systems wird zunächst eine fixe Emissionsobergrenze definiert, die für ein festgelegtes Gebiet, einen bestimmten Zeitraum und für teilnehmende Branchen in Form einer maximalen, meist verknappten, aber handelbaren Emissionsmenge gilt. Steigende Zertifikatspreise aufgrund der Verknappung der

2 Hinzu kommen die theoretischen Verhandlungslösungen nach Coase. Vgl. etwa G. Aberle: Transportwirtschaft, 5. Aufl., München 2009, S. 579-582; P. Michaelis: Ökonomische Instrumente in der Umweltpolitik: Eine anwendungsorientierte Einführung, Heidelberg 1996, S. 26 ff.

Dr. Sven Maertens ist wissenschaftlicher Mitarbeiter am Institut für Flughafenwesen und Luftverkehr des Deutschen Zentrums für Luft- und Raumfahrt (DLR) in Köln.

Dr. Janina Scheelhaase leitet dort die Gruppe Luftverkehrsökonomie.

Wolfgang Grimme, Dipl.-Kaufmann und Martin Jung, M.Sc., sind dort wissenschaftliche Mitarbeiter. 
Emissionsmenge geben den Marktteilnehmern einen Anreiz, zusätzliche Emissionen einzusparen. ${ }^{3}$ Je höher der Zertifikatspreis ausfällt, desto eher lohnt sich für einen Emittenten die Investition in neue, umweltfreundliche Produktionstechniken bei gleichzeitigem Verkauf nicht mehr benötigter Emissionsrechte. Um die Umwelteffektivität zu erhöhen, kann der Staat beispielsweise die Menge der Zertifikate im Zeitablauf oder die unentgeltliche Zertifikatsausgabe (Erstausstattung) reduzieren. Emissionen ohne Emissionsrechte werden sanktioniert. In sogenannten offenen Systemen besteht die Möglichkeit des Zukaufs von Emissionsrechten aus anderen Branchen bzw. des Verkaufs nicht benötigter Zertifikate dorthin. Bei einem geschlossenen System hingegen kann nur innerhalb der festgelegten Branche gehandelt werden.

Im Unterschied dazu erfolgt in einem Offsetting-System (Baseline\&Credit) keine absolute, politisch festgelegte Begrenzung (Cap) der Gesamtemissionsmenge. Vielmehr müssen Emissionsrechte für Emissionen, die über eine sogenannte Baseline hinausgehen, zugekauft werden. Diese Credits können durch Investition in Klimaschutzprojekte zur Treibhausgaseinsparung oder -speicherung erwirtschaftet werden und auf sogenannten Verpflichtungsmärkten (Compliance Markets) im Rahmen des Kyoto-Protokolls oder auf freiwilligen Märkten gehandelt werden. ${ }^{4}$ Hinzu kommen außerbörsliche Übertragungen insbesondere im Rahmen freiwilliger Klimakompensation über spezialisierte Dienstleister. Unabhängige Institutionen prüfen den Nutzen und die Qualität der Projekte und vergeben Zertifizierungen nach im Kyoto-Protokoll festgelegten Standards.

Im Ergebnis findet in einem Offsetting-System eine teilweise oder vollständig durch Offsets finanzierte Investition in klimafreundliche Projekte als Kompensation klimaschädlicher Emissionen statt. Auch das Instrument der Klimakompensation erlaubt es also, bei hohen Vermeidungskosten Emissionen durch die Finanzierung günstigerer Maßnahmen anderswo auszugleichen. Der „Nettoausstoß" von Treibhausgasen bleibt damit - wie auch bei einem Cap\&Trade-System - bei stringenter Anwendung gleich, weil es aus klimatologischer Sicht unerheblich ist, wo (auf der Erde) der Ausstoß an klimarelevanten Gasen bzw. die zugehörigen Einsparungen erfolgen. ${ }^{5}$

3 Zum Konzept der Umweltzertifikate vgl. grundlegend J. H. Dales: Pollution, Property and Prices, Toronto 1968.

4 Vgl. etwa J. Goodward, A. Kelly: Bottom Line on Offsets, World Resources Institute, August 2010, http://www.wri.org/publication/bottom-line-offsets (17.1.2017)

5 Vgl. hierzu z.B. J. Scheelhaase: Abgaben und Zertifikate als Instrumente zur $\mathrm{CO}_{2}$-Reduktion in der EG, München 1994.

\section{Emissionshandelssystem EU-ETS für den Luftverkehr}

Das 2005 zunächst für stationäre Quellen eingeführte Europäische Emissionshandelssystem zur Begrenzung der $\mathrm{CO}_{2}$-Emissionen ist das bislang größte internationale Cap\&Trade-System für Unternehmen zum Handel mit Emissionsrechten für Treibhausgase. ${ }^{6}$ Seit 2012 ist der Luftverkehr Teil des EU-ETS. Gemäß der Richtlinien 2008/101/EG und 2009/29/EG sollten seitdem die $\mathrm{CO}_{2}$-Emissionen aller Flüge von, nach und innerhalb der EWR-Staaten (EU, Norwegen, Island, Liechtenstein) grundsätzlich der Emissionshandelspflicht unterliegen. Der Geltungsbereich des EU-Emissionshandels wurde jedoch angesichts erheblicher Vorbehalte wichtiger Drittstaaten $^{7}$ im Zuge der sogenannten „Stopping-the-ClockEntscheidung" nachträglich durch Verordnung 421/2014/ EU zunächst für 2013 bis 2016 temporär eingeschränkt, weshalb gegenwärtig nur Flüge innerhalb des EWR wie folgt emissionshandelspflichtig sind (Reduced Scope): ${ }^{8}$

- Emissionsobergrenze (Cap) von 97\% der durchschnittlichen $\mathrm{CO}_{2}$-Emissionen der Jahre 2004 bis $2006 \mathrm{im}$ Jahr 2012 bzw. 95\% ab 2013;

- Kostenlose Zuteilung von Zertifikaten für $82 \%$ dieser Emissionsmenge auf Basis eines Benchmarks, der sich nach den durchschnittlichen Emissionen einer Fluggesellschaft in den Jahren 2004 bis 2006 sowie ihrer Verkehrsleistung 2010 richtet; Einhaltung einer „speziellen Reserve“ von 3\% des Caps zur kostenlosen Zuteilung an Neueinsteiger oder schnell wachsende Unternehmen und Versteigerung von Zertifikaten für die verbleibenden $15 \%$;

- Ausnahmen unter anderem für Flüge auf bestimmten Routen im Rahmen gemeinwirtschaftlicher Verpflichtungen, für sehr kleine Betreiber und Flugzeuge, sowie

6 Vgl. Europäische Kommission: Emissionshandelssystem der EU, https://ec.europa.eu/clima/policies/ets de (24.1.2017).

7 Beispielsweise verboten Senat und Kongress der USA US-Fluggesellschaften die Teilnahme am EU-ETS, vgl. Government Printing Office, European Union Emissions Trading Scheme Prohibition Act of 2011, 2012, http://www.gpo.gov/fdsys/pkg/PLAW-112publ200/pdf/PLAW-112publ200.pdf (24.1.2017). Offiziell wurde das „Stopping-the-Clock“ durch die EU allerdings mit positiven Entwicklungen auf der Ebene der Internationalen Zivilluftfahrtorganisation ICAO begründet. So heißt es in Absatz (3) der Vorbemerkungen zur Verordnung 421/2014/EU: „in order to sustain the momentum reached at the 38th Session of the ICAO Assembly in 2013 and facilitate progress at the upcoming 39th Session in 2016, it is desirable to temporarily consider the requirements set out in Directive 2003/87/EC of the European Parliament and of the Council ... to be satisfied for the period until 31 December 2016 in respect of flights to and from aerodromes in countries outside the European Economic Area (EEA)“.

8 Vgl. Deutsche Emissionshandelsstelle (DEHSt), https://www.dehst.de/ DE/Als-Betreiber-teilnehmen/Fuer-Luftfahrzeugbetreiber/fuer-luftfahrzeugbetreiber-node.html (28.4.2017). 
Abbildung 1

Fahrplan zur $\mathrm{CO}_{2}$-Emissionsreduzierung im Luftverkehr $\mathrm{CO}_{2}$-Emissionen

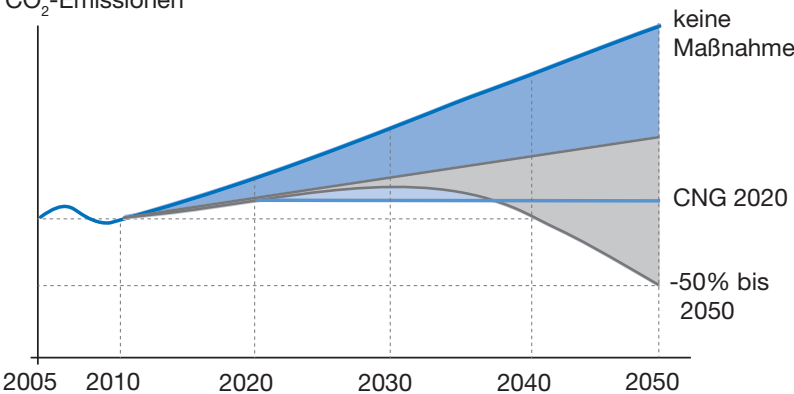

- Es werden keine Maßnahmen ergriffen

Einsatz bekannter Technologien, Prozesse und Infrastrukturen

- Einsatz von Bio-Kraftstoffen und alternativen Technologien

- $\mathrm{CO}_{2}$-freies Wachstum ab 2020 (CNG 2020)

- Brutto-Emissionen bei Einhaltung des IATA-Fahrplans

- Wirtschaftspolitische Maßnahmen greifen

Quelle: IATA, http://images.slideplayer.com/2/730793/slides/slide_11.jpg (17.1.2017).

für Sichtflüge, Rundflüge und Flüge im Rahmen humanitärer und ähnlicher Maßnahmen;

- Halboffenes System (Zukaufmöglichkeit von Emissionsrechten aus anderen Sektoren).

Zum Vorgehen von 2017 bis zur Einführung von CORSIA gibt es noch keine formalisierten Aussagen hinsichtlich der Einbeziehung oder Nicht-Einbeziehung von Flügen zwischen dem EWR und dem Rest der Welt.

\section{Der lange Weg zu CORSIA}

Das Ziel eines $\mathrm{CO}_{2}$-freien Wachstums des internationalen Luftverkehrs ab 2020 (CNG 2020) wurde auf der 37. Versammlung der ICAO 2010 in Resolution A37-19 beschlossen und auf der nachfolgenden Versammlung in Resolution A38-18 bekräftigt. ${ }^{9}$ Weiterhin wurde im Rahmen der 38. Versammlung der ICAO vereinbart, bis 2016 eine zusätzliche „global market-based measure“ (GMBM) zu entwickeln, um die Umsetzung dieses Ziels zu unterstützen. Dies ist erforderlich, da die Implementierung technischer und operativer Maßnahmen sowie ein verstärkter Einsatz von Biokraftstoffen alleine mindestens für den Zeitraum 2020 bis ca. 2037 nicht ausreichen werden, um das Ziel zu erreichen (vgl. Abbildung 1).

9 Vgl. International Civil Aviation Organization (ICAO): Resolution A37-19: Consolidated statement of continuing ICAO policies and practices related to environmental protection - Climate change, http://www.icao.int/ environmental-protection/37thAssembly/A37_Res19_en.pdf (20.1.2017); und dies.: Assembly Resolutions in Force, 4.10.2013, http://www.icao. int/Meetings/GLADs-2015/Documents/A38-18.pdf (20.1.2017).
Die Konkretisierung dieser marktbasierten Maßnahme erfolgte in der 39. Hauptversammlung durch Resolution A39-3 in Form des Offsetting-Systems CORSIA, ${ }^{10}$ dessen Anwendungsgebiet und Funktionsweise in Abbildung 2 dargestellt sind und wie folgt zusammengefasst werden können:

- CORSIA ist ein Klimakompensationssystem (Baseline \& Credit) für den internationalen Luftverkehr. Fluggesellschaften haben ihre Emissionen durch den Kauf von Offset-Zertifikaten auszugleichen, die unter anderem von Klimaschutzprojekten in anderen Sektoren herausgegeben werden.

- CORSIA beginnt 2020 und erstreckt sich über eine Pilotphase von 2021 bis 2023 sowie Phase 1 (2024 bis 2026) und Phase 2 (2027 bis 2035).

- Komplett von CORSIA ausgenommen sind zum einen kleine Emittenten (small emitters) mit weniger als 10000 t $\mathrm{CO}_{2}$ p.a., Flüge mit Flugzeugen von weniger als 5,7 thöchstzulässiger Startmasse, und humanitäre, medizinische und Löschflüge. Zudem fallen neue (und damit relativ stark wachsende) Fluggesellschaften für eine Dauer von maximal drei Jahren nicht unter CORSIA, es sei denn, sie erreichen bereits vorher einen individuellen Anteil an der weltweiten, internationalen Verkehrsleistung (gemessen in Revenue Ton Kilometers, RTK) von $0,1 \%$.

- Die Verpflichtung, Offsets zu halten, hängt nicht von Herkunft oder Größe des Betreibers, sondern nur von der beflogenen Strecke ab. Emissionsrechte müssen nur für „CORSIA-Emissionen“, d.h. Emissionen von internationalen Flügen zwischen teilnehmenden „CORSIA-Staaten“ nachgewiesen werden. Bis Ende 2026 ist die Teilnahme an CORSIA freiwillig und wird 67 Staaten umfassen (Stand vom 21.4.2017), deren Luftfahrzeugbetreiber rund $87,5 \%$ der internationalen RTK repräsentieren. ${ }^{11}$ Ein Ausstieg ist jährlich möglich.

- In Phase 2 muss jeder Staat an CORSIA teilnehmen, dessen Luftfahrzeugbetreiber 2018 mindestens 0,5\% der globalen, internationalen RTK repräsentieren, mit Ausnahme sogenannter Least Developed Countries

10 Vgl. ICAO: Resolutions adopted by the assembly, Oktober 2016, http://www.icao.int/Meetings/a39/Documents/Resolutions/a39_res_ prov_en.pdf (24.1.2017).

11 Vgl. ICAO.: Carbon Offsetting and Reduction Scheme for Internatio- Soll der nal Aviation (CORSIA), http://www.icao.int/environmental-protection/ aktuelle Pages/market-based-measures.aspx (21.4.2017). Zwischenzeitlich Stand nicht (Stand 20.7.2017) haben sich bereits 71 Staaten freiwillig zur Teilnahsentieren. Die zwei zusätzlichen Staaten sind in den Berechnungen im weiteren Verlauf dieses Artikels nicht berücksichtigt. 


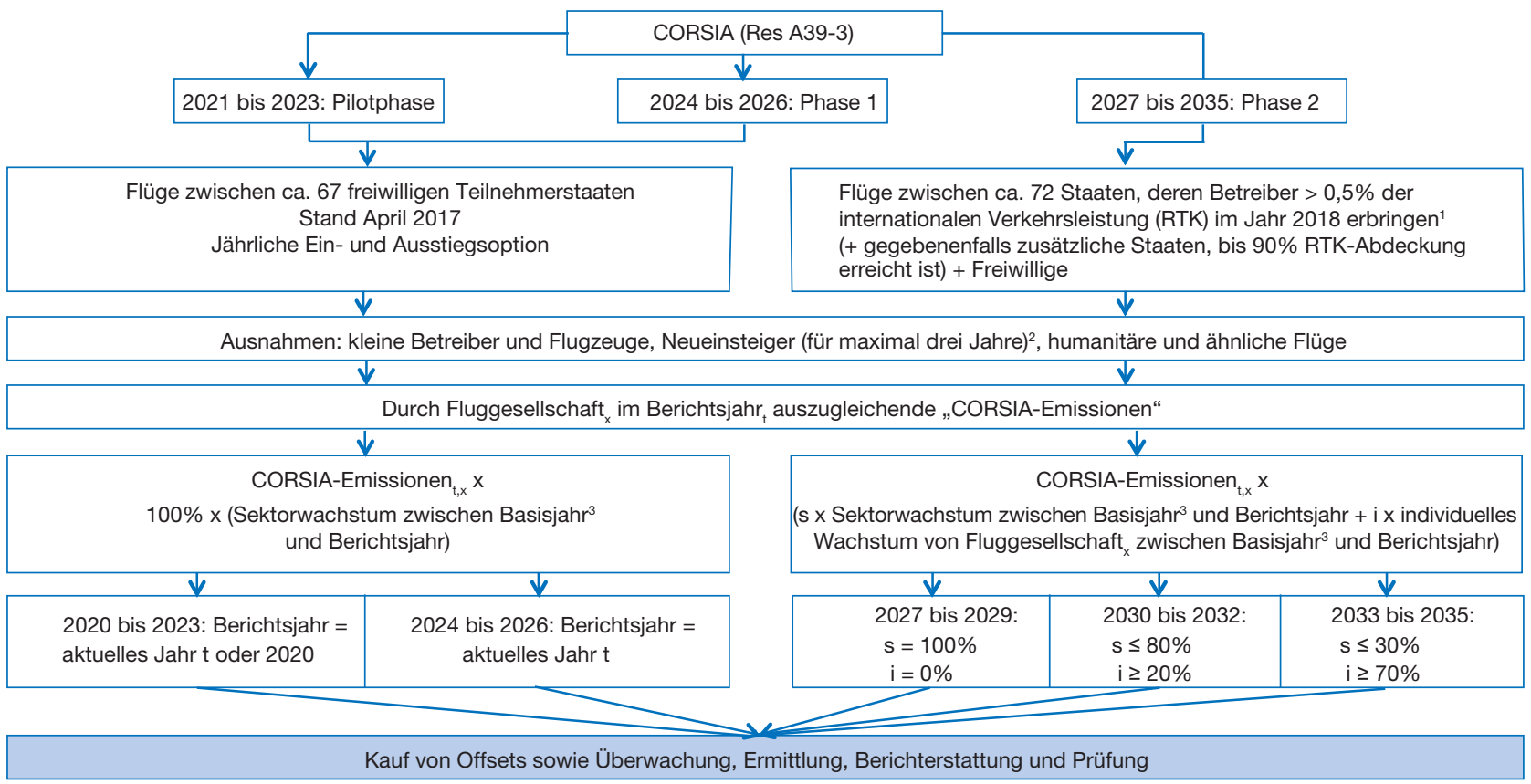

${ }^{1}$ Ausnahmen bilden Least Developed Countries (LDC), Small Island Developing States (SIDS) und Landlocked Developing Countries (LLDC), außer sie nehmen freiwillig teil. ${ }^{2}$ Sofern sie jeweils unter 0,1\% der weltweiten RTK bleiben. ${ }^{3}$ Mittelwert der Emissionen aus 2019 und 2020.

Quelle: eigene Abbildung.

(LDCs), Small Island Developing States (SIDS) und Landlocked Developing Countries (LLDCs). Eine freiwillige Teilnahme ist weiterhin möglich. Sofern die kumulierte Verkehrsleistung der Fluggesellschaften aller Teilnehmerstaaten unter $90 \%$ der internationalen RTK bleibt, sind auch die gemessen an der internationalen Verkehrsleistung nächst größerer Staaten miteinzubinden, bis die $90 \%$ erreicht sind. ${ }^{12}$

- Die exakte Menge an $\mathrm{CO}_{2}$-Emissionen, für die eine Fluggesellschaft in einem Berichtsjahr ab 2021 Offsets vorweisen muss, berechnet sich aus ihren individuellen CORSIA-Emissionen des Berichtsjahres multipliziert mit dem Wachstum aller CORSIA-Emissionen weltweit (Sektorwachstum) seit dem „Basisjahr“ (Mittelwert aus 2019 und 2020). In Phase 2 wird zunehmend auch das individuelle Wachstum der Fluggesellschaften mit herangezogen. Innerhalb der Pilotphase kann jeder teilnehmende Staat wählen, ob seine Fluggesellschaften bei der Berechnung der Abgabeverpflichtung die CORSIA-Emissionen des tatsächlichen Berichtsjahres oder die des Jahres 2020 anzusetzen haben.

12 Vgl. ICAO: Resolutions adopted by the assembly, a.a.O.

\section{Umwelteffektivität von CORSIA und EU-ETS}

Die Umwelteffektivität hängt beim Offsetting erstens von der festgelegten Menge der $\mathrm{CO}_{2}$-Baseline und von der zu kompensierenden $\mathrm{CO}_{2}$-Emissionsmenge ab. Zweitens ist es von großer Bedeutung, dass die Qualität der Offsets gesichert ist. Hat man sich für ein bestimmtes Instrumentarium entschieden, ist also die Umwelteffektivität dieser Maßnahme umso größer, je breiter sie angewendet wird.

Eine modellbasierte Schätzung der Umwelteffektivität von CORSIA im Vergleich zum EU-ETS erfolgte durch das am DLR-Institut für Flughafenwesen und Luftverkehr erstellte Emissionsberechnungstool 4D-RACE für den globalen Passagierverkehr 2016. Eine Modellierung des Emissionsbeitrags reiner Frachtflüge ist aufgrund einer unzureichenden Flugplandatengrundlage nicht möglich. Um das Luftverkehrswachstum und damit auch das Emissionswachstum langfristig abzubilden, wurden die „Central“-Szenarios der regionalisierten Luftverkehrsprognose der Forecasting and Economic Analysis Support Group (FESG) des ICAO Committee on Aviation Environmental Protection (CAEP) von 2014 auf die Modellierungsergebnisse für 2016 übertragen. In der Emissionsprognose wurde dabei für den Zeitraum 2017 bis 2039 eine 
Abbildung 3

Entwicklung der jährlichen globalen $\mathrm{CO}_{2}$-Emissionen des Passagierverkehrs

$\mathrm{CO}_{2}$-Emissionen in Mio. $\mathrm{t}$

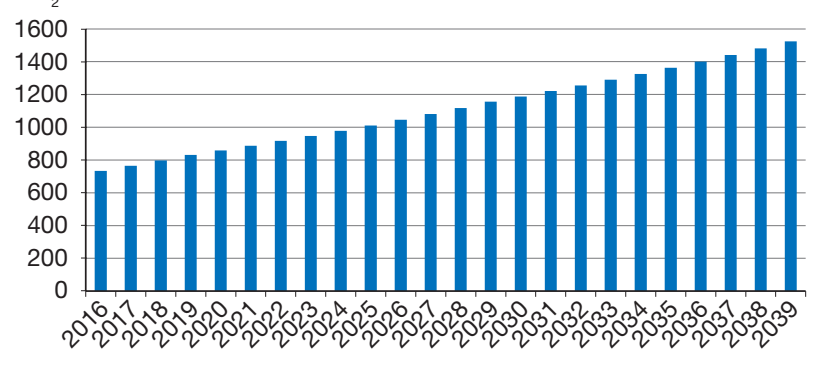

Jährliche globale Emissionen

Quelle: eigene Berechnungen mit dem DLR-Emissionstool 4D-Race für das ICAO FESG - „Central“--Prognoseszenarios.

durchschnittliche jährliche autonome Effizienzsteigerung von 1,2\% unterstellt - eine Größenordnung, die sich empirisch für die Entwicklung der vergangenen Jahrzehnte belegen lässt. Das erwartete durchschnittliche jährliche Emissionswachstum zwischen 2017 und 2039 auf globaler Ebene beträgt 3,2\%, sodass die jährlichen Emissionen von gegenwärtig 734 Mio. t auf schätzungsweise 1523 Mio. t $\mathrm{CO}_{2}$ ansteigen werden (vgl. Abbildung 3).

Eine durch CORSIA zu erzielende RTK-Abdeckung von 90\% bei der Ermittlung der ab Phase 2 teilnehmenden Staaten, bzw. die 87,5\%-ige Abdeckung durch die 67 freiwilligen Teilnehmerstaaten in den ersten zwei Phasen, klingen zunächst hoch. Diese beziehen sich aber einerseits nur auf den internationalen Luftverkehr, und zum anderen fallen Routen nur unter die Offset-Verpflichtung, wenn Start- und Zielland teilnehmen. Folglich entfallen derzeit (2016) nur etwa 42\% der globalen, auch durch Inlandsverkehre verursachten Gesamtemissionen des Passagierluftverkehrs auf Routen zwischen den 67 CORSIAStaaten.

Zudem unterliegen nicht sämtliche Emissionen dieser Flüge zwischen CORSIA-Staaten dem neuen System, sondern nur die durch das zusätzliche Wachstum des Sektors ab 2020 hervorgerufenen Emissionen. Ein großer Teil der jährlichen Emissionen, nämlich die 2020 erreichten „Sockelemissionen“, bleibt somit unreguliert. So wird 2021 nur eine relativ geringe Menge von 11,7 Mio. $\mathrm{t} \mathrm{CO}_{2}$ der Offset-Pflicht unterzogen und damit in anderen Emittenten-Bereichen und Projekten gemindert. Diese jährliche „Minderungsmenge“ wird jedoch im Zeitablauf durch das Emissionswachstum und die Einbeziehung weiterer Staaten immer weiter ansteigen und sich 2035 auf über 200 Mio. $\mathrm{CO}_{2}$ belaufen. Dies kann durchaus als umweltpolitischer Erfolg gewertet werden.

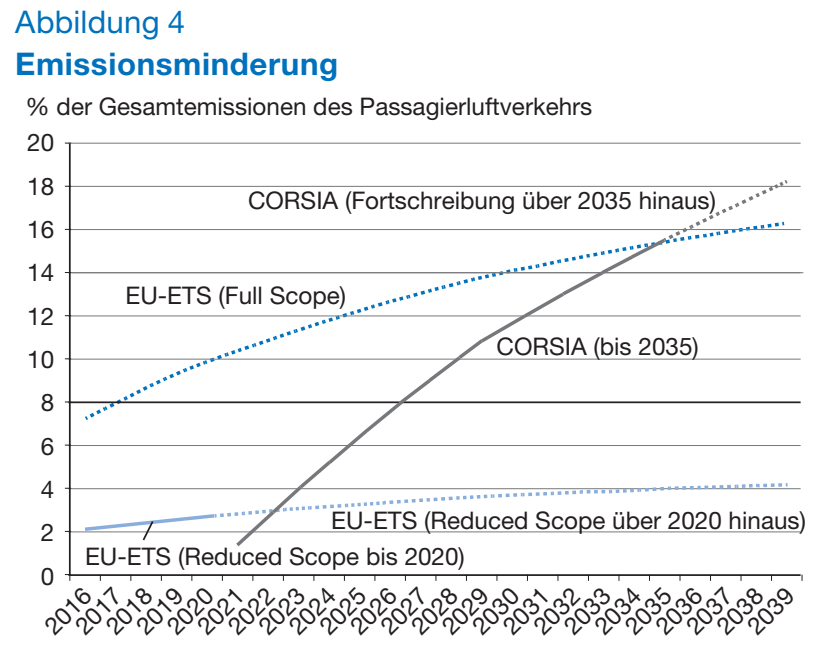

Die angezeigten Werte für CORSIA basieren aus Vereinfachungsgründen auch in Phase 2 nur auf den 67 freiwillig teilnehmenden CORSIA-Staaten (nach Stand April 2017).

Quelle: eigene Berechnungen mit dem DLR-Emissionstool 4D-Race für das ICAO FESG - „Central“-Prognoseszenarios.

Zum Vergleich: im aktuellen Reduced Scope des EU-ETS werden nur die Emissionen des Luftverkehrs im EWR reguliert, die 2016 rund 62,7 Mio. t $\mathrm{CO}_{2}$ betrugen und damit $8,5 \%$ der globalen $\mathrm{CO}_{2}$-Emissionen des Passagierluftverkehrs repräsentierten. Die Minderungsmenge aus dem EU-ETS ergibt sich aus den jährlichen Emissionen abzüglich des „Caps“ von 95\% der durchschnittlichen historischen Emissionen der Jahre 2004 bis 2006. Laut unseren Modellierungsergebnissen beträgt die auf den Passagierverkehr entfallende Obergrenze der Emissionen schätzungsweise 47,6 Mio. t. Daraus resultiert eine aktuelle Minderungsmenge von etwa 15,1 Mio. t $\mathrm{CO}_{2}$.

Die ursprüngliche Ausgestaltung des EU-ETS (Full Scope) umfasste alle im EWR startenden und landenden Flugzeuge auch im Drittstaatenverkehr. Hätte man diese Option auch 2016 politisch umgesetzt, wären mit etwa 220 Mio. t $\mathrm{CO}_{2}$ etwa $30 \%$ der globalen Luftverkehrsemissionen im Passagierverkehr in das EU-ETS einbezogen gewesen. Das Cap läge bei dieser Ausgestaltungsoption bei etwa 167 Mio. $t$, sodass sich die $\mathrm{CO}_{2}$-Minderungsmenge 2016 auf 53 Mio. $\mathrm{CO}_{2}$ beliefe.

In Abbildung 4 sind die aktuellen bzw. zukünftigen Anteile der durch marktbasierte Maßnahmen (CORSIA oder EUETS) geminderten Emissionen an den erwarteten Gesamtemissionen des Weltpassagierluftverkehrs dargestellt. Die durch CORSIA erzielte $\mathrm{CO}_{2}$-Minderung startet mit einem relativ kleinen Anteil von rund 1,4\%, steigt jedoch danach kontinuierlich an, da alle zusätzlichen Emissionen auf CORSIA-Routen ab 2021 durch Offsetting gemindert werden müssen. Selbst wenn man - wie in Abbildung 4 - 


\section{Abbildung 5}

Kumulierte Emissionsreduktion unter CORSIA und EU-ETS

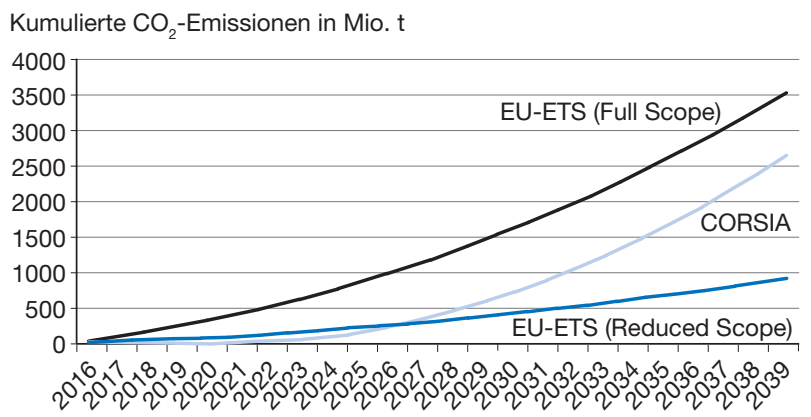

Quelle: eigene Berechnungen mit dem DLR-Emissionstool 4D-Race für das ICAO FESG - „Central“-Prognoseszenarios.

auch in Phase 2 nur die 67 freiwilligen CORSIA-Staaten zugrunde legt, erzielt man 2036 bereits einen Prozentsatz der Emissionsminderung an den Gesamtemissionen von über 16\%. Die $\mathrm{CO}_{2}$-Minderungsmenge im derzeitigen Reduced Scope des EU-ETS ist vergleichsweise gering, da im Intra-EWR-Verkehr nur ein geringer Teil der weltweiten Emissionen anfällt. Somit werden im Zeitablauf nur 2\% bis 4\% der globalen Luftverkehrsemissionen durch Offsetzertifikate und Emissionsrechte aus anderen Sektoren gemindert. Der ursprüngliche Plan der Europäischen Kommission, alle im EWR startenden und landenden Flüge in das EU-ETS einzubeziehen (Full Scope), weist kurzund mittelfristig die vergleichsweise höchste ökologische Effektivität auf. Bei dieser Ausgestaltungsoption würden aktuell knapp 8\% und bis 2039 gut 16\% der globalen $\mathrm{CO}_{2}$-Luftverkehrsemissionen des Passagierluftverkehrs reduziert. Die durch CORSIA geminderte Emissionsmenge würde das EU-ETS (Full Scope) erst Mitte der 2030er Jahre übersteigen.

Abbildung 5 zeigt zudem die kumulierte Emissionsreduktion im Zeitablauf ab 2016: Dies liegt bei CORSIA aus den genannten Gründen (Beginn erst im Jahr 2020, Regulierung nur der zusätzlichen jährlichen Emissionen ab diesem Zeitpunkt) zunächst, bis ca. 2027, unter der des EUETS im Reduced Scope, zieht dann aber vorbei. Die kumulierte Minderung, die bei Beibehaltung des Full Scopes im EU-ETS erzielbar gewesen wäre, wird indes bis 2039 nicht erreicht.

\section{Offene Fragen zum CORSIA-System}

Grundsätzlich problematisch bei der Anwendung von Umweltzertifikaten auf den Luftverkehr ist zudem die Frage, wie der Strahlungsantrieb (radiative forcing) bei der Festlegung der zu kompensierenden Emissionsmenge berücksichtigt werden soll. Hintergrund ist die Tatsache, dass der Luftverkehr eine Reihe klimarelevanter Emissionen verursacht, so z.B. $\mathrm{NO}_{x}, \mathrm{H}_{2} \mathrm{O}$, Kondensstreifen und Zirren sowie $\mathrm{CO}_{2}$. Umweltorganisationen argumentieren, dass die emittierte Menge aufgrund der höheren Wirkung in höheren Ozonschichten mit dem Faktor 2,7 (radiative forcing index, RFI) multipliziert werden sollte. ${ }^{13}$ Lee et al. betonen jedoch, dass ein pauschaler Ansatz des RFI als Multiplikator auf $\mathrm{CO}_{2}$-Emissionen des Luftverkehrs eine falsche Interpretation darstellt. ${ }^{14}$ Zudem hätte ein solcher Multiplikator den Nachteil, falsche Anreize zu setzen, weil für Investitionen in Technologien, die zu geringeren klimarelevanten Nicht- $\mathrm{CO}_{2}$-Emissionen führen, keine Anreize gesetzt werden. ${ }^{15}$ Das vom DLR im Auftrag des BMBF durchgeführte Projekt AviClim hat zur korrekten Berücksichtigung der wesentlichen klimarelevanten Emissionen des Luftverkehrs (auch in Emissionshandelssystemen) konkrete Vorschläge erarbeitet. ${ }^{16}$

Ein weiteres Problem kann die Verfügbarkeit einer ausreichenden Zahl an qualitativ genügenden Offsets darstellen, wenn mit dem Luftverkehr „Von jetzt auf gleich“ ein großer Nachfrager hinzukommt. Auch aus Sicht von Umweltorganisationen ist es hingegen vorteilhaft, dass als Ausgleich zu den Emissionen des Luftverkehrs konkret in Klimaprojekte investiert wird, während im EU-ETS Teile der Auktionserlöse auch in den normalen Staatshaushalt fließen können. ${ }^{17}$

\section{Wettbewerbspolitische Würdigung von CORSIA}

Grundsätzlich kann die Anwendung einer regulatorischen Maßnahme auf Teilmärkten zu Wettbewerbsverzerrungen auf dem Gesamtmarkt führen. Gleiches gilt für die Verwässerung des Systems durch Ausnahmen. Ähnlich wie schon im EU-ETS sind auch bei CORSIA Ausnahmen für kleine Betreiber (small emitters) relativ unkritisch, da diese als regionale Nischenanbieter im globalen Wettbewerbsumfeld kaum eine Rolle spielen.

13 Vgl. etwa Germanwatch: Fakten, die Sie nicht überfliegen sollten, http://germanwatch.org/klak/flug04.pdf (24.1.2017).

14 Vgl. D. S. Lee et al., a.a.O.

15 Vgl. P. Marbaix, A. Ferrone, B. Matthews: Inclusion of non- $\mathrm{CO}_{2}$ effects of aviation in the ETS: a summary, 28.8.2008, http://dev.ulb.ac.be/ ceese/ABC_Impacts/documents_abc/Note_to_stakeholders_aviation_nonco2.pdf (25.1.2017).

16 Vgl. J. Scheelhaase, K. Dahlmann, M. Jung, H. Keimel, H. Nieße, R. Sausen, M. Schaefer, F. Wolters: How to best address aviation's full climate impact from an economic policy point of view? - Main results from AviClim research project, Transportation Research Part D: Transport and Environment, Nr. 45, 2016, S. 112-125.

17 Vgl. auch L. Grießhaber: Emissionen begrenzen und Mittel für Klimafinanzierung generieren, Bepreisung von Emissionen im Flugverkehr international voranbringen, Positionspapier von Germanwatch, Berlin, Bonn, Februar 2013. 
Das EU-ETS hat aufgrund der geografischen Begrenzung auf Europa den Nachteil, dass Fluggesellschaften je nach Ausrichtung des Streckennetzes und Geschäftsmodells unterschiedlich stark belastet werden, auch wenn sie teilweise in Konkurrenz zueinander stehen. ${ }^{18}$ So fällt beispielsweise bei der Umsteigeverbindung einer griechischen Fluggesellschaft von Oslo über Athen nach Beirut das innereuropäische Flugsegment unter das EU-ETS, bei einer Konkurrenzverbindung einer türkischen Fluggesellschaft über Istanbul (Non-EWR) hingegen nicht. Durch die globale Ausrichtung von CORSIA wird dieser Nachteil zwar deutlich, aber nicht vollumfänglich aufgehoben. Dies liegt daran, dass weitgehende Ausnahmen für zwei Arten von Verkehren verbleiben:

1. Flüge von/zu/über Non-CORSIA-Staaten: Aufgrund des 0,5\%-RTK-Kriteriums ist damit zu rechnen, dass Staaten wie Ägypten, Vietnam, Sri Lanka, Pakistan, Kenia, Argentinien, Oman, Mauritius, die Seychellen oder die Malediven ${ }^{19}$ von CORSIA befreit sein werden. Dies reduziert nicht nur die Umwelteffektivität der Maßnahme, sondern führt auch zu Wettbewerbsverzerrungen zwischen „ähnlichen“ Flügen bzw. Destinationen (wie etwa den Kanarische Inseln vs. Ägypten, oder Thailand vs. Malediven). Durch die Definition der von CORSIA ausgenommenen Staaten alleine auf Basis der Verkehrsleistung 2018 können zudem sogar Staaten bis zum Ende der 2. Phase von CORSIA ausgenommen bleiben, deren Carrier nach 2018 ein enormes Wachstum verzeichnen.

2. Inlandsverkehre: Die Ausklammerung sämtlicher Inlandsflüge im CORSIA-System ist in wettbewerblicher Hinsicht besonders bei flächenmäßig großen Staaten wie China, der Russischen Föderation oder den USA relevant. So fällt eine direkt bediente Strecke wie Frankfurt/Los Angeles vollständig unter CORSIA, während bei einem Routing von Frankfurt über einen Hub an der US-Ostküste nach Los Angeles nur das Transatlantiksegment, nicht aber der inneramerikanische Anschlussflug reguliert wird. Bei einer Fluggesellschaft mit einem hohen Anteil an Inlandsverkehr unterliegt daher ein geringerer Anteil der Verkehrsleistung und damit am Umsatz dem CORSIA-System als bei einem Anbieter mit einem hohen Anteil an Auslandsverkehr. Diese wettbewerbsverzerrende Wirkung von CORSIA ist systemimmanent, da die ICAO als UN-Organisation ausschließlich für den internationalen Luftverkehr zu-

18 Vgl. etwa J. Scheelhaase, W. Grimme, M. Schaefer: The inclusion of aviation into the EU emissions trading scheme - impacts on competition between European and non-European network airlines, Transportation Research Part D: Transport and Environment, Nr. 15, 2010, S. 14-25.

19 Vgl. ICAO: Civil Aviation: 2012 International RTK by State of Air Operator Certificate (AOC), last updated 24.9.2013. ständig ist. Abhilfe könnte hier nur die freiwillige Anwendung der CORSIA-Regelungen auch auf den inländischen Luftverkehr schaffen.

Eine weitere Wettbewerbsverzerrung kann dadurch entstehen, dass in den ersten beiden Phasen von CORSIA auf die Emissionsmenge aller Fluggesellschaften ausschließlich ein einheitlicher Multiplikator, nämlich das Sektorwachstum angewendet wird, um die Menge an nötigen Offsets zu bestimmen. Dies hat zur Folge, dass auch nicht (mehr) wachsende Fluggesellschaften Offsetts erwerben müssen.

\section{Transaktionskosten der Maßnahme}

Ein administrativer Aufwand bei Fluggesellschaften, Behörden und Prüfstellen, wie er bereits aus dem EU-ETS bekannt ist, wird auch in CORSIA entstehen, und dies in geografisch weitaus größerer Breite. Zum einen müssen die Fluggesellschaften für alle CORSIA-Routen ihre Verkehrsleistung und ihren Kerosinverbrauch protokollieren und berichten. Dies trifft nicht nur auf Fluggesellschaften aus den anfangs 67 freiwillig teilnehmenden Staaten zu, sondern auch auf Fluggesellschaften aus Drittstaaten, die auf CORSIA-Routen operieren. Hinzu kommen behördliche Überwachung- und Prüfaktivitäten der Heimatstaaten dieser Fluggesellschaften und Aktivitäten von Prüfstellen, sowie - zumindest anfangs - entsprechende Schulungsaktivitäten. Emissionen im Rahmen des EU-ETS werden hingegen von Betreibern auch aus Drittstaaten an zuständige EU-Staaten gemeldet, sodass behördliche Überwachung- und Prüfaktivitäten außerhalb Europas bislang nicht nötig sind.

Zum anderen besteht die Notwendigkeit eines globalen RTK-Reportings sämtlicher Fluggesellschaften weltweit im Jahr 2018. Dies gilt selbst für Staaten, die aufgrund relativ geringer Aktivitäten ihrer Fluggesellschaften von CORSIA befreit sein werden. Diese Verpflichtung ist nichts Neues auf ICAO-Ebene. Jedoch offenbaren Berichte der ICAO für vergangene Jahre, dass bislang ein großer Teil von Staaten keine entsprechenden Daten gemeldet hat und dies betrifft nicht nur Entwicklungsländer. ${ }^{20}$ Weiterhin müssen auch nicht von CORSIA abgedeckte Staaten vereinfachte Berichtspflichten erfüllen, damit die Gesamtemissionen ermittelbar sind. Schließlich wird im Rahmen von CORSIA eine Überwachung der Güte der gehandelten Offsets erfolgen müssen, was weitere administrative Prozesse nach sich ziehen wird.

Eine allerdings rechtlich wohl schwer umsetzbare zentralisierte CORSIA-Verwaltung könnte die aus den genann-

20 Vgl. ebenda 
ten Aktivitäten resultierenden administrativen Kosten teilweise senken. Alternativ wären bei einem grundsätzlich anderen Design der marktbasierten Maßnahme niedrigere Transaktionskosten denkbar gewesen, wie etwa bei einer Upstream-Lösung, bei der Mineralölfirmen Offsets für Kerosinlieferungen vorweisen müssen. Dies hätte Berichterstattungs-, Überwachungs- und Prüfaktivitäten mit einer viel kleineren Akteurszahl bedeutet.

\section{Fazit}

Gegenstand dieses Beitrags war eine Vorstellung und kritische Analyse der im Herbst 2016 auf Ebene der Internationalen Zivilluftfahrtorganisation verabschiedeten marktbasierten Maßnahme CORSIA zu Begrenzung von klimarelevanten Emissionen des internationalen Luftverkehrs. Dabei wurde auch auf die wesentlichen Unterschiede zum derzeitigen Einbezug des Luftverkehrs in den Emissionshandel im Europäischen Wirtschaftsraum eingegangen.

Sowohl das EU-ETS als auch CORSIA zielt darauf ab, ökonomische Anreize zur Vermeidung klimarelevanter Emissionen des Luftverkehrs zu setzen. Dem EU-ETS unterliegen derzeit nur rund 8,5\% der weltweiten Emissionen des Luftverkehrs, da Verbindungen zwischen Europa und dem Rest der Welt entgegen ursprünglicher Pläne bis auf Weiteres ausgenommen sind. Zudem fällt die effektive Minderungsmenge mit rund 2,1\% noch deutlich geringer aus, da von den besagten 8,5\% Emissionsanteil nur das Wachstum zwischen dem „Cap“ (95\% des Durchschnitts der Emissionen 2004 bis 2006) und den Emissionen des betrachteten Jahres durch Offsetzertifikate oder Emissionsrechte aus anderen Sektoren ausgeglichen werden muss.

Die umweltökonomische Bewertung des CORSIA-Systems fällt ambivalent aus. Einerseits wird der internationale Luftverkehr zwischen nahezu allen bedeutenden Luftverkehrsnationen einbezogen und nicht nur der Verkehr innerhalb des EWR. Andererseits ist das Ziel eines $\mathrm{CO}_{2}^{-}$ neutralen Wachstums ab 2020 wenig ambitioniert und führt nicht zu einer absoluten Senkung der Luftverkehrsemissionen im historischen Vergleich, denn die Emissionen steigen kumulativ weiter an.

Schätzungen auf Basis einer DLR-Emissionsprognose zeigen, dass für den Luftverkehr in seiner ursprünglichen Ausgestaltung durch CORSIA kurz- und mittelfristig eine geringere Umwelteffektivität zu erwarten ist als durch das EU-ETS. In der langen Frist, d.h. ab 2035 steigt die ökologische Wirksamkeit von CORSIA hingegen an: So trägt CORSIA $2035 \mathrm{zu}$ einer Reduktion der $\mathrm{CO}_{2}$-Emissionen des Luftverkehrs um etwa 200 Mio. $t$ bei. Gleichwohl steigen die klimarelevanten Emissionen im Luftverkehr kumulativ weiter.

Um die Umwelteffektivität marktbasierter Maßnahmen im Luftverkehr nach 2020 zu steigern, könnten z.B. in der EU Möglichkeiten diskutiert werden, Teile des EU-ETS beizubehalten. Dies ist insbesondere im Hinblick darauf zu erwägen, dass beim Übergang vom EU-ETS zu CORSIA das im EWR zwischen 2004 bis 2006 und 2020 realisierte Emissionswachstum und die gesamten Inlandsverkehre nicht mehr einer marktbasierten Maßnahme unterliegen würden. Unseren Schätzungen zufolge belaufen sich alleine die $\mathrm{CO}_{2}$-Emissionen des Inlandsverkehrs in den EWR-Staaten 2016 auf mindestens 13 Mio. t $\mathrm{CO}_{2}$.

In CORSIA begründete Wettbewerbsverzerrungen zwischen Fluggesellschaften sind im Gegensatz zum EUETS angesichts der hohen weltweiten Abdeckung moderat, auch wenn vereinzelt Routen bzw. Destinationen mit und ohne CORSIA-Belastung miteinander konkurrieren. Allerdings hat die Anwendung eines einheitlichen Multiplikators in den ersten zwei Phasen zur Folge, dass auch nicht (mehr) wachsende Fluggesellschaften ihre Emissionen ausgleichen müssen.Aufgrund des DownstreamAnsatzes mit hohen Überwachungs-, Berichterstattungsund Verifizierungsaktivitäten auf weltweiter Ebene ist CORSIA für den Luftverkehr vermutlich noch transaktionskostenintensiver als das EU-ETS.

Title: Climate Protection in Air Transport by Market-based Measures - from EU ETS to CORSIA

Abstract: To address the carbon footprint of global air transport, two important CO2 trading schemes for aviation are in force, or will be in the future: The EU Emissions Trading Scheme (EU ETS) for aviation from 2012, and CORSIA (Carbon Offsetting and Reduction Scheme for International Aviation) as agreed at International Civil Aviation Organization (ICAO) level in October 2016. The authors analyze and compare both reduction schemes from environmental and economic perspectives. JEL Classification: Q580, H23, L93 